\title{
Tratamento dos transtornos alimentares: perfil dos pacientes e desfecho do seguimento
}

\section{Treatment of eating disorders: patient profile and outcome tracking}

\author{
Marina Garcia Manochioํㄹ ${ }^{1}$ Pamela Gabrielle Reis ${ }^{1}$, Helena de Souza Luperi ${ }^{1}$, Rosane Pilot Pessa ${ }^{2}$, \\ Felícia Bighetti Sarrassini ${ }^{1}$ \\ 1- Universidade de Franca - UNIFRAN, Franca, SP, Brasil. \\ 2- Universidade de São Paulo - Escola de Enfermagem de Ribeirão Preto, Ribeirão Preto, SP, Brasil.
}

marina.manochio@unifran.edu.br

Palavras-chave:

Transtornos da alimentação e da ingestão de alimentos;

Bulimia;

Anorexia .

Keywords:

Feeding and eating

disorders;

Nervous Bulimia;

Anorexia Nervosa.

\section{RESUMO}

Objetivo: traçar o perfil e o desfecho do tratamento dos pacientes atendidos por um serviço especializado, a fim de buscar indicadores de um bom prognóstico, e por meio de uma amostragem quantitativa e qualitativa, possibilitar reflexões sobre o serviço existente. Método: foram analisados 185 prontuários de pacientes atendidos com diagnósticos divididos em anorexia nervosa restritiva, anorexia nervosa bulímica e bulimia. Após levantamento de dados, foram realizados testes estatísticos, Qui-Quadrado, Análise de variância e o pós-teste Bonferroni, com $95 \%$ $(\mathrm{p}<0,05)$ de confiabilidade. Resultados: houve prevalência do sexo feminino (92\%), sendo que a maior incidência ocorreu entre os 10 e 19 anos, apresentando, em sua maioria, anorexia nervosa bulímica. Dos prontuários analisados, $58 \%$ dos pacientes abandonaram o tratamento, $20 \%$ tiveram alta, $10 \%$ permanecem em atendimento e os outros $10 \%$ dividem-se entre encaminhamento, desligamento e óbitos. Conclusão: concluiu-se que os pacientes atendidos pelo serviço são, em sua maioria, mulheres jovens apresentando anorexia nervosa. Também foi encontrada elevada taxa de abandono ao tratamento. Menor tempo de doença e início precoce do tratamento são indicadores de bom prognóstico. Estudos como este são importantes para auxiliar profissionais na busca de recursos para melhores resultados nos acompanhamentos desses quadros clínicos, a fim de minimizar o alto índice de abandono e promover melhores resultados de recuperação clínica.

\section{ABSTRACT}

Objective: to trace the profile and treatment outcome of patients treated by a specialized service in order to seek indicators of a good prognosis, and through a quantitative and qualitative sampling to obtain reflections on the existing service. Method: we analyzed 185 medical records of patients treated by the service with diagnostics divided into restrictive anorexia nervosa, anorexia nervosa and bulimia. After data collection, it were applied the statistical tests, chi-square, analysis of variance and Bonferroni post-test, with $95 \%(\mathrm{p}<0.05)$ significance. Results: the higher prevalence was of female subjects (92\%), and the highest incidence was between 10 and 19 years, mainly of individuals with bulimic anorexia nervosa. The records analyzed indicated that $58 \%$ of patients abandoned the treatment, $20 \%$ were discharged, $10 \%$ remain in medical care and the other $10 \%$ are divided between forwarding, shutdown and deaths. Conclusion: in this study, it was concluded that the patients enrolled in the service are mostly young women having anorexia nervosa. It was also found a high dropout rate to treatment. Shorter disease and early treatment are good prognostic indicators. Studies like this one are important to help professionals in search of resources for better results in the follow-up of these medical conditions in order to minimize the high dropout rate and promote better results in clinical recovery. 


\section{INTRODUÇÃO}

Os transtornos alimentares (TA) são quadros psiquiátricos caracterizados por grave alteração do comportamento alimentar ou da absorção de alimentos, comprometendo a saúde física ou o funcionamento psicossocial, podendo originar prejuízos biológicos e psicológicos e aumento da morbidade e mortalidade. ${ }^{1-6}$ A interação desses componentes com o quadro familiar e sociocultural justifica sua etiologia multifatorial.,

Os sistemas classificatórios dos transtornos mentais, Diagnostic and Statistical Manual, V edition (DSM-V), e a Classificação Internacional de Doenças, $10^{\text {a }}$ edição (CID-10) ${ }^{9}$ ressaltam a anorexia nervosa (AN) e a bulimia nervosa (BN) como duas entidades nosológicas. Ambas apresentam como psicopatologia comum o medo de engordar. ${ }^{10}$

$\mathrm{O}$ início do quadro clínico na $\mathrm{AN}$, geralmente se dá com a restrição de grupos alimentares, eliminando aqueles que julga mais calóricos. Essa restrição alimentar aumenta progressivamente, com diminuição do número de refeições, podendo evoluir drasticamente, até o jejum. Existem dois tipos de apresentação da AN: o restritivo (AN-R) e o bulímico (AN-B). No primeiro, os pacientes utilizam comportamentos restritivos associados à dieta. $\mathrm{Na}$ AN-B ocorrem episódios de compulsão alimentar, seguidos de métodos compensatórios, como vômitos auto induzidos e o uso de laxantes e diuréticos. ${ }^{3,9}$ Devido a esses fatores, as principais complicações clínicas na AN são decorrentes da desnutrição causada pela ingestão alimentar muito abaixo das necessidades de macro e micronutrientes ocasionando a queima de reservas de gordura e massa muscular, com perda de peso acentuada, carências nutricionais importantes e diminuição da taxa metabólica basal. As alterações metabólicas são semelhantes àquelas encontradas no estado de inanição e é comum ocorrer hipercolesterolemia, ocasionada, na maioria das vezes, por perda grave da massa de gordura corporal. A lipólise encontra-se aumentada, além do retardo na remoção das partículas de lipoproteína de baixa densidade. ${ }^{11-14}$

$\mathrm{Na} \mathrm{BN}$, as alterações do quadro clínico são decorrentes da alta ingestão energética (episódios bulímicos, denominados binge eating - BE) e da distribuição desequilibrada dos nutrientes, ocasionadas pelodescontrolealimentarepela práticademecanismos compensatórios, como vômitos e abuso de laxantes e/ou diuréticos. Frequentemente, são encontrados distúrbios hidroeletrolíticos, principalmente hipocalemia, alterações gastrintestinais, endócrinas, hematológicas e no sistema imunológico, com redução nos mecanismos de defesa e alterações na matriz cerebral. Podem ocorrer diminuição no crescimento e problemas dentários, com aumento da frequência do desenvolvimento de cáries e perda dos dentes, além da elevação dos triglicérides plasmáticos e do comprometimento muscular e cardíaco. ${ }^{3,12,15,16}$

O manejo no tratamento dos TA sofreu uma série de avanços nas últimas décadas; no entanto, continua sendo árduo, prolongado, de resultados duvidosos e, muitas vezes, insatisfatórios. Isso se deve, em parte, às condições inerentes à doença que, na sua complexidade e rebeldia, representa um desafio a qualquer esforço terapêutico. Por outro lado, existem os mecanismos de negação acionados pelos pacientes e seus familiares no sentido de negar a gravidade, quando não a própria existência do problema, dificultando a adesão ao tratamento. Por esse motivo, o número de abandonos ao tratamento é alto. ${ }^{17}$ Dentre os principais objetivos do tratamento ambulatorial dos TA, podem ser destacadas: reabilitação nutricional, cessação de comportamentos para alterações de peso, melhora nos comportamentos alimentares e no estado psicológico e emocional. ${ }^{18}$

Conhecer os indicadores de bom e mau prognóstico dos TA possibilita determinar com maior precisão a intensidade e o tipo de tratamento. Nos estudos sobre $\mathrm{AN}$ e $\mathrm{BN}$, os fatores mais frequentes de bom prognóstico são: idade menor de início da doença, menor número de hospitalizações, tempo pequeno de doença antes do tratamento e sintomatologia leve a moderada. Os fatores de mau prognóstico incluem: duração prolongada da doença, início tardio da patologia, presença de sintomas bulímicos nos casos de AN, peso inicial muito baixo, relações familiares problemáticas prévias ao TA e estado marital (casada), sintomatologia mais severa, frequência aumentada de vômitos no início do quadro, flutuações extremas de peso, impulsividade, baixa autoestima, conduta suicida e transtornos comórbidos, como uso de substâncias no início da patologia. ${ }^{19}$

Miller e Golden ${ }^{20}$ estimam que o prognóstico de recuperação de um TA varia de acordo com o diagnóstico, a população de estudo, e duração do acompanhamento/tratamento. Pessoas com AN têm maior incidência de recaída e taxa de mortalidade 
mais elevada em comparação com as pessoas com diagnóstico de outros TA.

Os dados literários são escassos sobre o desfecho do tratamento de pacientes que recebem alta dos serviços. Sabe-se que o critério para este fim é um assunto bastante polêmico quando se trata de TA, sendo que o estado nutricional adequado não representa, sozinho, critério para alta. ${ }^{17}$

Devido à escassez de dados na literatura que auxiliem os profissionais no manejo técnico e, ainda, pessoal desses pacientes, é possível observar grande dificuldade na atuação do tratamento de TA. Observase, ainda, que nos serviços do Brasil, há escassez de divulgação da prevalência dos TA e dos resultados do tratamento, pois estes não apresentam dados sobre abandono, recaída e alta, entre outros, deixando clara a necessidade dessa atividade científica. Estudos dessa natureza exigem disponibilidade de recursos financeiros e utilização de instrumentos diagnósticos específicos e validados para a detecção desses quadros. Essa é considerada uma tarefa árdua e complexa, o que dificulta a realização de levantamentos epidemiológicos de base populacional com amostra representativa da população brasileira. ${ }^{7,21}$

O objetivo do presente estudo foi traçar o perfil e o desfecho do tratamento dos pacientes atendidos em um serviço especializado, a fim de buscar indicadores de bom prognóstico, e por meio de amostragem quantitativa e qualitativa, possibilitar reflexões sobre o serviço existente.

\section{MÉTODO}

Trata-se de estudo descritivo, observacional, transversal com delineamento quanti-qualitativo, desenvolvido em um serviço especializado para tratamento de TA, o Grupo deAssistência a Transtornos Alimentares (GRATA), localizados no Hospital das Clínicas da Faculdade de Medicina de Ribeirão Preto da Universidade de São Paulo (HCFMRPUSP). O GRATA é um programa do Ambulatório de Nutrologia do HCFMRP-USP, filiado à Universidade de São Paulo (USP), em atividade desde 1982. Atualmente, é composto por médicos nutrólogos, psiquiatras, nutricionistas, psicólogos e estagiários, além de enfermeiros e terapeutas ocupacionais, no caso de internação. ${ }^{22,23}$

Os participantes do presente estudo foram todos os pacientes atendidos por esse serviço, independentemente da modalidade de atendimento (ambulatorial e internação integral), desde sua criação, em 1982, até o primeiro semestre de 2011. Após a aprovação do Comitê de Ética em Pesquisa da Universidade de Franca (UNIFRAN) (Ref.n 0010/1), os dados foram coletados a partir do levantamento dos prontuários dos pacientes com diagnóstico de $\mathrm{AN}$ e BN, sendo o primeiro subdividido em AN-R e AN-B. Com base nesses critérios, realizou-se revisão de todos os arquivos disponíveis (prontuário físico, digitalizado e microfilmado), com o intuito de verificar o seguimento do paciente no momento inicial do tratamento (primeiro atendimento), e em seu último retorno ao serviço (último atendimento). Desde a sua fundação, o GRATA já assistiu aproximadamente 240 indivíduos com algum tipo de TA. ${ }^{24}$

O critérioutilizado parainclusão dosindivíduos na pesquisa foi, em primeiro lugar, ter passado por atendimento no GRATA e ter como diagnóstico AN-R, AN-B ou BN. Além desses critérios, foram inclusos os indivíduos cuja situação posterior ou atual ao atendimento se enquadravam em uma das seguintes situações: em seguimento no momento da coleta de dados ${ }^{\mathrm{a}}$, abandono ${ }^{\mathrm{b}}$, alta $^{\mathrm{c}}$, encaminhamento para outros serviços especializados ${ }^{\mathrm{d}}$, desligamento por inassiduidade do paciente ou óbito.

Os critérios utilizados para exclusão de pacientes da pesquisa foi a impossibilidade de observação de alguns prontuários, devido a problemas no sistema do hospital, dificuldades em ler prontuários antigos e/ ou com informações escassas. Além desses critérios, foram retirados pacientes que apresentaram TA não especificado.

Foram utilizadas, posteriormente à coleta de dados, as variáveis sociodemográficas: sexo, idade, estado civil, profissão e procedência, além das variáveis relacionadas à saúde e diagnostico, alteração de peso, evolução e tempo de tratamento.

\footnotetext{
a Assim que o serviço realiza o chamado caso novo, o paciente está admitido no serviço, ou seja, está em seguimento.

${ }^{\mathrm{b}}$ Acontece quando o paciente desiste do acompanhamento.

${ }^{\text {c } Q u a n d o ~ o ~ p a c i e n t e ~ n a ̃ o ~ s e ~ e n c o n t r a ~ m a i s ~ e m ~ e s t a d o ~ c l i ́ n i c o-n u t r i c i o n a l ~}$ preocupante ou, ainda, quando conseguem lidar melhor com seus comportamentos alimentares.

${ }^{d}$ Quando a procedência dos pacientes está fora da XVIII Região de Saúde do Estado de São Paulo ou, ainda, quando há a presença indicação para outros serviços.

${ }^{\text {e }}$ Realizado quando o paciente apresenta três faltas consecutivas, ou cinco faltas alternadas, sem justificação comprovada das mesmas, ou, ainda, caso a presença de familiares ou representantes familiares tenha sido definida no plano de atendimento inicial e os mesmos tiverem as mesmas faltas acima citadas.
} 
Para análise dos resultados obtidos, os testes de análise estatística utilizados foram: Qui-Quadrado, Análise de Variância e pós-teste de Bonferroni, com confiabilidade de $95 \%(\mathrm{p}<0,05)$.

\section{RESULTADOS}

Após análise dos prontuários desses pacientes, foram incluídos, para este estudo, 185 indivíduos que apresentavam um dos diagnósticos citados. Vinte e três prontuários foram excluídos. Foram encontrados dois prontuários de pacientes já atendidos pelo serviço que interromperam o tratamento e retornaram posteriormente, evento denominado de recaída, porém não inclusos nos testes estatísticos, por falta de registros de tempo (data do último atendimento da primeira vez que passaram pelo serviço) e justificativa da saída do mesmo.

Pôde-se perceber que a maior parte dos atendimentos do GRATA foram realizados em pacientes do sexo feminino, em sua maioria, jovens, estudantes e solteiras. Torna-se oportuno mencionar que o número reduzido de pacientes do sexo masculino não permitiu identificar a possível associação entre a variável sexo e as categorias de TA (teste QuiQuadrado: $\mathrm{p}>0,05$ ).

Como apresentado na Tabela 1 , os resultados evidenciaram maior incidência de casos com diagnóstico de AN-B e AN-R, dados também observados no sexo masculino. A maior incidência de TA ocorreu na faixa etária de 10 a 19 anos, com prevalência de AN-R; seguida pela faixa de 20 a 29 anos, prevalecendo AN-B seguida pela BN; e, posteriormente, pela faixa de 30 a 39 anos, em que também prevaleceu a AN-B. Pacientes com 40 anos ou mais, a AN-R e BN obtiveram resultados equivalentes (Tabela 1).

a maior parte dos pacientes que já estiveram ou ainda estão em atendimento apresentou ganho ou recuperação de peso.

Como já era esperado, em relação à localização geográfica dos pacientes, foi constatado que a maioria

Tabela 1 - Características sociodemográficas, desfecho e tempo de tratamento dos 185 pacientes atendidos pelo serviço, segundo diagnóstico (Ribeirão Preto, 2011).

\begin{tabular}{|c|c|c|c|c|c|c|c|c|c|c|}
\hline \multirow{2}{*}{$\begin{array}{l}\text { Variáveis } \\
\text { Sexo }\end{array}$} & \multirow[t]{2}{*}{ Tempo $^{a}$} & \multirow[t]{2}{*}{ Desvio Padrão } & \multicolumn{2}{|c|}{ ANR } & \multicolumn{2}{|c|}{ ANB } & \multicolumn{2}{|c|}{ BN } & \multicolumn{2}{|c|}{ Total } \\
\hline & & & $n$ & $\%$ & $\bar{n}$ & $\%$ & $n$ & $\%$ & $\bar{n}$ & $\%$ \\
\hline Masculino & & & 8 & 4,32 & 6 & 3,24 & 0 & 0 & 14 & 7,54 \\
\hline Feminino & & & 63 & 34,05 & 67 & 36,22 & 41 & 22,2 & 171 & 92,46 \\
\hline Total & & & 71 & 38,37 & 73 & 39,46 & 41 & 22,2 & 185 & 100 \\
\hline \multicolumn{11}{|l|}{ Idade } \\
\hline 10-19 anos & & & 51 & 27,6 & 37 & 20 & 16 & 8,65 & 104 & 56,22 \\
\hline 20-29 anos & & & 12 & 6,5 & 26 & 14,05 & 18 & 9,73 & 56 & 30,27 \\
\hline 30-39 anos & & & 7 & 3,8 & 8 & 4,32 & 6 & 3,24 & 21 & 11,35 \\
\hline 40 anos ou mais & & & 1 & 0,54 & 2 & 1,08 & 1 & 0,54 & 4 & 2,16 \\
\hline Total & & & 71 & 38,44 & 73 & 39,45 & 41 & 22,16 & 185 & 100 \\
\hline \multicolumn{11}{|l|}{ Evolução } \\
\hline Abandono & 20,65 & 28,62 & 40 & 21,62 & 44 & 23,78 & 24 & 12,97 & 108 & 58,38 \\
\hline Alta & 43,54 & 27,10 & 18 & 9,73 & 12 & 6,49 & 7 & 3,78 & 37 & 20 \\
\hline Desligamento & 38,24 & 33,18 & 3 & 1,62 & 3 & 1,62 & 2 & 1,08 & 8 & 4,32 \\
\hline Encaminhamento & 54,26 & 87,36 & 2 & 1,08 & 3 & 1,62 & 5 & 2,7 & 10 & 5,4 \\
\hline Seguimento & 64,34 & 85,71 & 6 & 3,24 & 10 & 5,4 & 2 & 1,08 & 18 & 9,73 \\
\hline Óbito & 20,57 & 28,19 & 2 & 1,08 & 0 & 0 & 0 & 0 & 2 & 1,08 \\
\hline Recaída & - & - & 0 & 0 & 1 & 0,54 & 1 & 0,54 & 2 & 1,08 \\
\hline Total & 241,6 & & 71 & 38,38 & 73 & 39,46 & 41 & 22,16 & 185 & 100 \\
\hline
\end{tabular}

${ }^{a}$ média do tempo de tratamento (em meses).

Segundo o teste Qui-Quadrado, houve significância quando observada a relação entre faixa etária e diagnóstico, o que demonstra relação positiva entre as idades no período da adolescência com o desenvolvimento da doença; ou melhor, que a idade apresentada pelos pacientes que já passaram por atendimento, ou que ainda estão em seguimento, tenha relação ao diagnóstico apresentado. Em geral, 
era procedente de Ribeirão Preto e região, como mostra a Tabela 2. Este fato pode explicar o alto índice de abandono em todos diagnósticos, decorrente da falta de adesão ao tratamento, falta de aceitação da doença ou, ainda, pela distância a ser percorrida até o atendimento.

Do total de pacientes com $\mathrm{AN}$ e $\mathrm{BN}$, em relação ao desfecho apresentado, observou-se que a maior parte abandonou o tratamento, em segundo lugar estão os pacientes que receberam alta, sendo a maioria do grupo AN-R, seguido pela AN-B. Em seguida, estão os pacientes em seguimento durante a coleta, a maioria apresentou diagnóstico de AN-B, seguido de AN-R e BN. Por último, ficaram os índices de desligamento e encaminhamento (Tabela 2). As ocorrências de desligamento se deram em quantidades equivalentes para AN-R e AN-B; para BN foi menor. Ao mesmo tempo, somente pacientes com AN-R foram a óbito. Metade daqueles encaminhados para outros serviços apresentaram diagnóstico de $\mathrm{BN}$.

Tabela 2 - Características sociodemográficas dos 185 pacientes atendidos pelo serviço (Ribeirão Preto, 2011).

\begin{tabular}{lcc}
\hline Variável & Frequência & \% \\
\hline Profissão $^{\mathbf{a}}$ & 106 & 57 \\
Estudante & 37 & 20 \\
Com ocupação & 12 & 6 \\
Do Lar/Sem ocupação & & \\
Procedência $^{\mathrm{b}}$ & 140 & 76 \\
Ribeirão Preto e região $_{\text {Interior de SP }}$ & 26 & 14 \\
Cidades de MG & 15 & 8 \\
Outros estados & 2 & 1 \\
\hline
\end{tabular}

${ }^{a}$ considerado sem ocupação aqueles pacientes sem trabalho com renda; sem informação: 30 pacientes; bsem informação: dois pacientes. Foram definidas cidades da região de Ribeirão com distância de até $100 \mathrm{~km}$, interior de SP: cidades com mais de 100km de Ribeirão e outros estados: DF e TO; Pacientes com procedência na cidade de Ribeirão Preto: 76

Ao se analisar cada tipo de diagnóstico em relação ao seu desfecho, não foi encontrada diferença estatística significativa pelo teste Qui-Quadrado, ou seja, otipo de TA(AN-R,AN-BeBN)não estáassociado com o desfecho (alta, abandono, desligamento, óbito, seguimento ou encaminhamento), o que significa que o tipo de desfecho encontrado, neste caso, não dependeu do diagnóstico.

Quando analisado o tempo de tratamento por desfecho, encontrou-se diferença estatística pelo teste da Análise de Variância, ou seja, o tempo de tratamento pode estar associado ao desfecho, o que indica que o tempo é um fator que pode influenciar no tipo de evolução. Para esta análise, foi realizado o Teste de Bonferroni, e os resultados apontam que o tempo de tratamento contribui para alta e abandono. Deve-se lembrar que a influência do tempo de tratamento para as demais evoluções pode não ter apresentado significância estatística por existirem poucos pacientes com os demais desfechos. O tempo de tratamento ainda apresentou diferença estatística quando comparado com o diagnóstico, o que significa que este influenciou o tempo de tratamento pelo Teste de Análise de Variância. Com o teste Bonferroni, observou-se que o tempo de tratamento se diferencia para AN-R e AN-B: o paciente com AN-B teve tempo menor quando comparado com aquele com AN-R. Quanto à $\mathrm{BN}$, não houve significância estatística.

\section{DISCUSSÃO}

Para Sancho et al. ${ }^{25}$, as mulheres mostram maior incidência e prevalência de TA que os homens, o mesmo foi encontrado nos estudos de Appolinário ${ }^{26}$, Hoek $^{27,28}$ e Teixeira et al. ${ }^{29}$, que afirmam que cerca de $90 \%$ dos pacientes são do sexo feminino, o que corrobora os achados do presente estudo, pois $92 \%$ dos pacientes que passaram pelo serviço são do sexo feminino. Para Feitlich et al. ${ }^{30}$, os principais fatores etiológicos da anorexia são o sexo (se apresenta nove vezes maior em mulheres) e a idade (tendo início, geralmente, na adolescência). Em estudo realizado por Alves et al. ${ }^{31}$, a média de idade das adolescentes com AN foi de 14,1 anos, dados que se equiparam com os do presente estudo, pois a idade de prevalência de início de tratamento no GRATA apresentou-se na faixa de 10 à 19 anos, seguida pela de 20 a 29 anos. Em estudo de revisão, Qian et al. ${ }^{32}$ também encontraram grande prevalência de TA em mulheres quando comparadas a homens (relação 4:2), sendo o maior diagnóstico $\mathrm{AN}$, que vem aumentando significativamente nos últimos anos.

Estudos com pacientes bulímicas mostraram que o início do desenvolvimento do transtorno se dá na adolescência, até aproximadamente 20 anos. ${ }^{9}$ $\mathrm{Na}$ presente pesquisa, observou-se algo semelhante, pois a maioria dos pacientes com $\mathrm{BN}$ esteve na faixa etária de 20 a 29 anos. Hay $^{33}$ avaliou 102 mulheres diagnosticadas com $\mathrm{BN}$ e 67 com história de $\mathrm{AN}$, todas com idade entre 16 e 35 anos, enquanto que, 
neste estudo, a maioria dos pacientes na faixa etária de 10 a 39 anos apresentava diagnóstico de AN em comparação com o número menos expressivo de casos clínicos de pacientes com BN.

Agras et al..$^{34}$ afirmaram que a AN se inicia com a restrição da dieta, evoluindo ao longo do tempo para a compulsão alimentar e purgação, ou seja, AN-B em mais de $50 \%$ dos casos, dados estes comprovados também nos achados desta pesquisa, já que, dos 144 pacientes com $\mathrm{AN}, 39,5 \%$ apresentaram $\mathrm{AN}-\mathrm{B}$.

$\mathrm{Na}$ literatura, poucos dados são encontrados sobre o prognóstico dessas síndromes devido, principalmente, às dificuldades metodológicas. Ribeiro et al. ${ }^{17}$ citam um estudo em que Nemiah identificou na sua amostra $70 \%$ de cura ou melhora clínica dos seus casos, $22 \%$ de cronicidade, o que não é muito diferente no estudo de Agras et al. ${ }^{34}$, em que $20 \%$ tornaram-se crônicos e, ainda, $8 \%$ de casos de mortalidade. Este último item bem maior do que o encontrado nesta casuística $(1,08 \%)$. Pinzon e Nogueira ${ }^{19}$ relataram que as taxas de mortalidade podem variar entre $5 \%$ e $20 \%$, e que, na maioria das vezes, ocorre por complicações da própria AN.

Reas et al. $^{35}$ realizaram um estudo, cujos resultados revelaram que $72,7 \%$ dos participantes se recuperaram durante o tratamento/seguimento. $\mathrm{O}$ bom resultado foi associado com a menor duração do quadro, definido como o tempo entre o início dos sintomas e a primeira intervenção. Em vários estudos analisados por Abreu e Cangelli Filho ${ }^{36}$, cerca de $50 \%$ das pacientes se "recuperaram", ou seja, restabeleceram o peso, e os comportamentos alimentares se normalizaram, além da regularização da menstruação. No presente estudo, somando as altas de todos os diagnósticos, cerca de $20 \%$ dos pacientes tiveram alta do serviço pela melhora do quadro clínico apresentado. Porém, não foi possível dizer em que situação estes se encontram hoje, já que não se teve acesso a essas informações.

A BN parece ter evolução mais favorável que a $\mathrm{AN}$, embora as pesquisas com $\mathrm{BN}$ ainda tenham pouco tempo de seguimento. Os estudos indicam índices de recuperação total entre $50 \%$ e $70 \%$, de acordo com os seus períodos de acompanhamento após manejo terapêutico. Herzog et al. ${ }^{37}$ relataram que a taxa de recuperação total das mulheres com BN foi significativamente maior que a de mulheres com AN. Na presente casuística, o número de pacientes com alta em $\mathrm{AN}$ foi maior que em BN. Este resultado pode ser explicado pelo maior número de pacientes com AN (considerando AN-R e AN-B) quando comparado com BN. Todavia, as taxas de recaída situaram-se em torno de $30 \%$ a $50 \%$. Paralelamente, as taxas de mortalidade da BN ficaram entre $0,3 \%^{38}$ e $3 \%$, de acordo com a gravidade dos casos clínicos das amostras analisadas. ${ }^{19}$ Desses achados, o único que se aproxima dos deste estudo é o índice de óbito, pois dentre os pacientes bulímicos, não houve índice de mortalidade. Freitas ${ }^{39}$ acredita que a baixa taxa de mortalidade na $\mathrm{BN}$ se dá devido ao menor período de seguimento comparado à AN. A taxa de mortalidade aumenta à medida que aumenta o seguimento desses estudos, além disso, se consideradas as perdas de seguimento dos estudos mais longos, a taxa de mortalidade por BN pode estar subestimada.

Ainda sobre pacientes com BN, Hoste et al..$^{40}$ demonstraram taxa de abandono de $11 \%$, dados que se aproximam dos achados desta pesquisa, que encontrou taxa de abandono de $13 \%$ dos pacientes com diagnóstico de BN. Entretanto, outros estudos encontraram índices amplos de abandono, tanto para AN quanto $\mathrm{BN}$, variando entre $11 \%$ e $45 \% .40-42$

Carvalho ${ }^{43}$, em estudo realizado em um serviço especializado em TA, observou taxa de 19,42\% de abandono em 103 pacientes com $\mathrm{AN}$ e BN, taxa relativamente baixa quando comparada à do presente estudo, que apresentou $58 \%$ de abandono ao tratamento. Em contrapartida, Alvarenga e Larino $^{44}$ relataram elevada taxa de abandono. Das 39 pacientes que iniciaram o tratamento, $24(61,54 \%)$ não terminaram, resultados estes que se aproximam aos deste estudo. Agras et al. ${ }^{34}$, encontraram 26\% de desistência do tratamento, enquanto que Bacaltchuk e Hay ${ }^{45}$ identificaram, em estudo de revisão, que a taxa de abandono em curto prazo foi de $30 \%$, taxa relativamente alta por ser um estudo de curto prazo, ressaltando que a presente pesquisa foi realizada ao longo de 29 anos.

Em relação ao total de pacientes que abandonaram o tratamento, o número de AN (AN$\mathrm{R}$ e $\mathrm{AN}-\mathrm{B})$ foi 3,5 vezes maior em relação à $\mathrm{BN}$. Este fato pode ser explicado devido ao número bem maior de pacientes com AN. Porém, em vários outros estudos que investigaram ambos os TA, verificou-se que pacientes com $A N$, em especial $A N-B$, são os que mais abandonam o tratamento, chegando à taxa de $60 \%$ de desistência. ${ }^{43,42,46,47}$

Pinzon e Nogueira ${ }^{19}$ relataram que, depois de 
intervenções terapêuticas, as taxas de recuperação completa de AN ficaram em torno de $50 \%$, resultado distante do encontrado no presente estudo pois somando-se as altas de AN-R e AN-B, encontrouse $16,22 \%$ em relação aos 144 pacientes com AN. Wagner et al. ${ }^{48}$ afirmaram que, em longo prazo, $50 \%$ dos indivíduos com TA se recuperaram, porém, não foi possível dizer se este é realmente um bom resultado, já que não se tem informações seguintes à alta. No presente estudo, este índice ficou um pouco abaixo do encontrado pelos autores, sendo que $20 \%$ dos pacientes até hoje atendidos tiveram alta do serviço. No presente estudo, somente pacientes com diagnóstico de AN-R foram a óbito. Em estudo de revisão, os autores encontraram altas taxas de mortalidade em ambos os diagnósticos, porém com incidência muito maior entre os pacientes anoréxicos. Os autores ainda associaram essa maior taxa de mortalidade às condições em que o paciente iniciou o tratamento e ao prognóstico do mesmo ${ }^{49}$.

Sobre recaída de cronicidade, não há consenso sobre o significado desses termos. Estudos sugerem adotar um tempo mínimo sem que o paciente apresente sintomas, e após este período haja o reaparecimento de um ou mais sintomas apresentados no início do tratamento. ${ }^{39}$ Outro estudo aponta que o risco de recaída no seguimento surgiu apenas quatro anos após o início do tratamento, com isto os autores sugeriram um período de seguimento maior para verificar esse desfecho. ${ }^{38}$ No presente estudo foram encontrados dois casos de recaída entre os pacientes com diagnóstico de AN-B e BN, porém dados incompletos nos prontuários em relação ao tempo não permitiram concluir a real situação desses pacientes. Além deste fato, é importante ressaltar que não se sabe a situação atual de pacientes que obtiveram alta ou abandonaram o serviço, visto que estes não mais procuraram o serviço.

Esses dados encontrados e já apresentados em inúmeros estudos só tendem a reforçar a importância dos esforços terapêuticos, que devem sempre se basear em programas multidisciplinares que promovam a reabilitação nutricional com base na reeducação alimentar e no acompanhamento clínico, psicológico, comportamental e familiar, que visam abranger todos os intrigantes aspectos somáticos, psicológicos, familiares e sociais que se apresentam nos casos dos distúrbios alimentares. ${ }^{40}$

\section{CONCLUSÃO}

Assim, concluiu-se que a maioria dos pacientes, que já passaram pelo GRATA, apresentaram AN e que a incidência de TA é significativamente maior no sexo feminino entre os 10 e 29 anos. Observou-se, ainda, elevada taxa de abandono, principalmente de pacientes com AN, seguida por alta e valores menos expressivos nos demais desfechos. Em relação ao prognóstico, pode-se observar neste estudo e em estudos já citados, que a menor duração do quadro clínico antes da procura pelo serviço pode ocasionar em melhor evolução e desfecho. A idade precoce de início da doença também mostra maior rapidez na procura por tratamento.

Devido à escassez de estudos que auxiliem no manejo desses pacientes por profissionais, o tratamento de TA se torna muito trabalhoso e nem sempre traz resultados satisfatórios. Estudos como este são importantes para auxiliar profissionais na busca de recursos para melhores resultados nos acompanhamentos desses quadros clínicos a fim de minimizar o alto índice de abandono e promover melhores resultados de recuperação clínica.

\section{REFERÊNCIAS}

1. Alvarenga MS, Philippi ST. Estrutura, padrão, consumo e atitude alimentar: conceitos e aplicações nos transtornos alimentares. In: Alvarenga M, Scagliusi FB, Philippi ST. Nutrição e transtornos alimentares: uma visão nutricional. Barueri: Manole; 2011. doi: 10.1176/foc.3.4.546.

2. Dunker KLL, Phillipi ST. Hábitos e comportamentos alimentares de adolescentes com sintomas de anorexia nervosa. Revista Nutr. 2003;16(1):51-60. doi: 10.1590/S141552732003000100006.

3. Borges NJBG, Sicchieri JMF, Ribeiro RPP, Marchini JS, Dos Santos JE. Transtornos alimentares - Quadro clínico. Medicina (Ribeirão Preto) 2006;39(3):340-8.

4. Björk T, Ahlström G. The patient's perception of having recovered from an eating disorder. Health Care Women Int. 2008;29(8):926-44. doi: 10.1080/07399330802269543.

5. Costas RAN, Lamela DJPV, Costa LG. Teoria e eficácia da terapia comportamental dialética na bulimia nervosa e no transtorno da compulsão alimentar periódica. J Bras Psiquiatr. 2009;58(2):122-7. doi: 10.1590/S0047-20852009000200010.

6. Ferreira JES, Veiga GV. Confiabilidade (teste-reteste) de um questionário simplificado para triagem de adolescentes com comportamentos de risco para transtornos alimentares em estudos epidemiológicos. Rev Bras Epid 2008;11(3):393-401. doi: 10.1590/S1415-790X2008000300006.

7. Ribeiro RPP, Santos MA. Transtornos alimentares: evidências clínicas e científicas. In: Almeida, SS. et al. Psicobiologia 
do comportamento alimentar. Rio de Janeiro: Ed. Rubio. 2013. 8. Nunes MA, Appolinario JC, Galvão NA, Coutinho W. Transtornos alimentares e obesidade. 2nd Ed. Porto Alegre: Artmed; 2006.

9. American Psychiatric Association (APA). Diagnostic and statistical manual of mental disorders. 5th Ed. Arlington: American Psychiatric Association. 2013.

10. Claudino AM, Borges MBF. Critérios diagnósticos para os transtornos alimentares: conceitos em evolução. Rev Bras Psiquiatr 2002;24(Supl.3):7-12. doi: 10.1590/S151644462002000700003.

11. Rigaud, D. Anorexia nervosa: a model of malnutrition. Ann Med Interne (Paris) 2000;151(7):549-55.

12. Saito MI, Silva LEV. Adolescência: prevenção e risco. In: Cereser MG, Cordás TA. (orgs.). Transtornos alimentares: anorexia e bulimia. São Paulo: Atheneu. 2001. p. 269-76.

13. Weinbrenner T, Züger M, Jacoby GE, Herpertz S, Liedtke R, Sudhop T, Gouni-Berthold I, Axelson M, Berthold HK. Lipoprotein metabolism in patient with anorexia nervosa: a case-control study investigating the mechanism leading to hypercolesterolaemia. Br J Nutr 2004;91(6):959-69. doi: 10.1079/BJN20041151.

14. Zwaan M, Aslam Z, Mitchell JE. Research on energy expenditure in individuals with eating disorders: a review. Int J Eat Disord. 2002;32(2):127-34. doi: 10.1002/eat.10074.

15. Kerem NC, Katzman DK. Brain structure and function in adolescents with anorexia nervosa. Adolesc Med 2003;14(1):109-18.

16. Philippi ST, Alvarenga MS. Alimentação saudável: princípios e recomendações. In: Alvarenga MS, Philippi ST. (orgs.). Transtornos alimentares: uma visão nutricional. Barueri: Manole. 2004. p. 21-37.

17. Sicchieri JMF, Santos MA, Santos JE, Ribeiro RPP. Avaliação nutricional de portadores de transtornos alimentares: resultados após alta hospitalar. Ciênc Cuid Saúde 2007;6(1):68-75. doi: 10.4025/cienccuidsaude.v6i1.4975.

18. Fagundes U, Oliva CAG. Avaliação e tratamento das complicações médicas. In: Claudino AM, Zanella MT. (eds.). Guias de medicina ambulatorial e hospitalar: Transtornos alimentares e obesidade. $1^{a}$ Ed. São Paulo: Manole. 2005. p. 119-26.

19. Pinzon V, Nogueira FC. Epidemiologia, curso e evolução dos transtornos alimentares. Rev Psiquiatr Clín 2004;31(4):15860. doi.org/10.1590/S0101-60832004000400004.

20. Miller CA, Golden NH. An introduction to eating disorders: Clinical presentation, epidemiology and prognosis. Nutr Clin Pract 2010 25(2):110-15. doi: 10.1177/0884533609357566.

21. Manochio MG. O perfil e a atuação do nutricionista no tratamento dos transtornos alimentares. 2009. 129f. Dissertação (Mestrado). Escola de Enfermagem de Ribeirão Preto da Universidade de São Paulo. Ribeirão Preto. 2009. doi. 10.11606/D.22.2009.tde-18082009-123504.

22. Santos JE. Grata: nossa história, trabalho e desafios. Medicina (Ribeirão Preto). 2006;39(3):323-6.

23. Palma RFM, Santos JE, Ribeiro RPP. Hospitalização integral para tratamento dos transtornos alimentares: a experiência de um serviço especializado. J Bras Psiquiatr 2013; 62(1):31-7. doi: 10.1590/S0047-20852013000100005.

24. Souza APL, Pessa RP. Tratamento dos transtornos alimentares: fatores associados ao abandono. J Bras Psiquiatr 2016;65(1):60-7. doi: 10.1590/0047-2085000000104.
25. Sancho C, Arija MV, Asorey O, Canals J. Epidemiology of eating disorders: A two year follow up in an early adolescents school population. Eur Child Adolesc Psychiatry 2007;16(8):495-504. doi: 10.1007/s00787-007-0625-0.

26. Appolinário JC. Transtorno do comer compulsivo. In: Nunes MA, Appolinário JC, Abuchaim ALA, Coutinho W. (orgs.). Transtornos alimentares e obesidade. Porto Alegre: Artes Médicas. 1998. p. 40-6.

27. Hoek HW. Review of the prevalence and incidence of eating disorders. Int J Eat Disord 2003;34(4):383-96. doi: 10.1002/ eat. 10222 .

28. Hoek HW. Incidence, prevalence and mortality of anorexia nervosa and other eating disorders. Curr Opin Psychiatry 2006;19(4):389-94. doi: 10.1097/01.yco.0000228759.95237.78. 29. Teixeira PC, Costa RF, Matsudo SMM, Cordás TA. A prática de exercícios físicos em pacientes com transtornos alimentares. Rev Psiquiatr Clín 2009;36(4):145-52. doi.org/10.1590/ S0101-60832009000400004.

30. Fleitlich BW, Larino MA, Cobelo A, Cordás TA. Anorexia nervosa na adolescência. J Pediatr. 2000;76(Supl. 3):S323-9.

31. Alves E, Calvo MCM, Neves J, Vasconcelos FAG. Prevalência de sintomas de anorexia nervosa e insatisfação com a imagem corporal em adolescentes do sexo feminino do Município de Florianópolis, Santa Catarina, Brasil. Cad. Saúde Pública 2008;24(3):503-12. doi.org/10.1590/S0102311 X2008000300004.

32. Qian J, Hu Q, Wan Y, Li T, Wu M, Ren Z, Yu D. Prevalence of eating disorders in the general population: a systematic review. Shanghai Arch Psychiatry 2013;25(4):212-23. doi: 10.3969/j.issn.1002-0829.2013.04.003.

33. Hay PJ. Epidemiologia dos transtornos alimentares: estado atual e desenvolvimentos futuros. Rev Bras Psiquiatr 2002;24(Supl. 3):13-7. doi: 10.1590/S151644462002000700004.

34. Agras WS, Brandt HA, Bulik CM, Dolan-Sewell R, Fairburn CG, Halmi KA, Herzog DB, Jimerson DC, Kaplan AS, Kaye WH, le Grange D, Lock J, Mitchell JE, Rudorfer MV, Street LL, Striegel-Moore R, Vitousek KM, Walsh BT, Wilfley DE. Report of the National Institutes of Health workshop on overcoming barriers to treatment research in anorexia nervosa. Int J Eat Disord 2004;35(4):509-21. doi: 10.1002/eat.10261

35. Reas DL, Williamson DA, Martin CK, Zucker NL. Duration of illness predicts outcome for bulimia nervosa: A long-term follow-up study. Int J Eat Disorders 2000;27(4):428-34. doi: 10.1002/(SICI)1098-108X(200005)27:4<428::AID-EAT7>3.0. $\mathrm{CO} ; 2-\mathrm{Y}$

36. Abreu CN, Cangelli Filho R. Anorexia nervosa e bulimia nervosa: abordagem cognitivo-construtiva de psicoterapia. Rev Psiquiatr Clín 2004;31(4):177-83. doi.org/10.1590/S010160832004000400010.

37. Herzog DB, Dorer DJ, Keel PK, Selwyn SE, Ekeblad ER, Flores AT, Greenwood DN, Burwell RA, Keller MB. Recovery and relapse in anorexia and bulimia nervosa: a 7.5-year follow-up study. J Am Acad Child Adolesc Psychiatry1999;38(7):829-37. doi: 10.1097/00004583-199907000-00012.

38. Keel PK, Mitchell JE. Outcome in bulimia nervosa. Am J Psychiatry 1997;154(3):313-21. doi: 10.1176/ajp.154.3.313.

39. Freitas SR. Curso e prognóstico dos transtornos alimentares. In: Nunes MA, Appolinario JC, Galvão AL, Coutinho W (orgs.). 
Transtornos alimentares e obesidade. São Paulo: Artmed. 2008. p. $105-16$.

40. Hoste RR, Zaitsoff S, Hewell K, le Grange D. What can dropouts teach us about retention in eating disorder treatment studies? Int J Eat Disord 2007;40(7):668-71. doi: 10.1002/ eat. 20421 .

41. Watson HJ, Fursland A, Byrne S. Treatment engagement in eating disorders: who exits before treatment? Int J Eat Disord 2013;46(6):553-9. doi: 10.1002/eat.22085.

42. Carter O., Pannekoek L., Fursland A., Allen KL., Lampard AM., Byrne SM. Increased wait-list time predicts dropout from outpatient enhanced cognitive behaviour therapy (CBT-E) for eating disorders. Behav Res Ther 2012;50(7-8):487-92. doi: 10.1016/j.brat.2012.03.003.

43. Carvalho LSL. Fatores de risco para o abandono do tratamento hospitalar para transtornos alimentares. 2013. 139f. Dissertação (Mestrado). Faculdade de Medicina da Universidade de São Paulo. São Paulo. 2013.

44. Alvarenga M, Larino MA. Terapia nutricional na anorexia e bulimia nervosas. Rev Bras Psiquiatr 2002;24(3):39-43. doi. org/10.1590/S1516-44462002000700009.

45. Bacaltchuk J, Hay P. Tratamento da bulimia nervosa: síntese das evidências. Rev Bras Psiquiatr 1999;21(3):184-7. doi: 10.1590/S1516-44461999000300012.

46. Bandini S, Antonelli G, Moretti P, Pampanelli S, Quartesan R, Perriello G. Factors affecting dropout in outpatient eating disorder treatment. Eat Weight Disord 2006;11(4):179-84.

47. Fassino S, Pierò A, Tomba E, Abbate-Daga G. Factors associated with dropout from treatment for eating disorders: a comprehensive literature review. BMC Psychiatry 2009; 9:67. doi: 10.1186/1471-244X-9-67.

48. Wagner A, Barbarich-Marsteller NC, Frank GK, Bailer UF, Wonderlich SA, Crosby RD, Henry SE, Vogel V, Plotnicov K, McConaha C, Kaye WH. Personality traits after recovery from eating disorders. Int J Eat Disord 2006;39(4):276-84. doi: 10.1002/eat.20251

49. Arcelus J, Mitchell AJ, Wales J, Nielsen S. Mortality rates in patients with anorexia nervosa and other eating disorders: a meta-analysis of 36 studies. Arch Gen Psychiatry 2011;68(7):72431. doi: 10.1001/archgenpsychiatry.2011.74.

50. Yager J, Devlin MJ, Halmi KA, Herzog DB, Mitchell JE, Powers PS, Zerbe KJ. Practice guideline for the treatment of patients with eating disorders. Focus 2005;3(4):546-55. doi. org/10.1176/foc.3.4.546.

Recebido em:01/11/2017

Como citar: MANOCHIO, Marina Garcia et al. Tratamento dos transtornos alimentares: perfil dos pacientes e desfecho do seguimento. Revista Interdisciplinar de Promoção da Saúde, Santa Cruz do Sul, v. 1, n. 1, jan. 2018. ISSN 2595-3664. Disponível em: <https://online.unisc.br/seer/index.php/ripsunisc/article/view/11946>. Acesso em: 01 janeiro 2018. doi:http://dx.doi. org/10.17058/rips.v1i1.11946 